\title{
Isolated complex III deficiency
}

INSERM

\section{Source}

INSERM. (1999). Orphanet: an online rare disease and orphan drug data base. Isolated complex III deficiency. ORPHA:1460

Isolated complex III deficiency is a rare, genetic, mitochondrial oxidative phosphorylation disorder characterized by a wide spectrum of clinical manifestations rang ing from isolated myopathy or transient hepatopathy to severe multisystem disorder (that may include hypotonia, failure to thrive, psychomotor delay, cardiomyopathy, encephalopathy, renal tubulopathy, hearing impairment, lactic acidosis, hypoglycemia and other signs and symptoms). 